\title{
Research progress of the clinicopathologic features of lung adenosquamous carcinoma
}

This article was published in the following Dove Press journal:

OncoTargets and Therapy

\begin{abstract}
Jing Wangl,*
Yanling Wangl,*

Mengting Tong ${ }^{2}$

Hongming Pan'

$\mathrm{Da} \mathrm{Li}$
\end{abstract}

'Department of Medical Oncology, Sir Run Run Shaw Hospital, Zhejiang University School of Medicine, Hangzhou 3l00I6, Zhejiang, China;

${ }^{2}$ Second Department of Oncology, The Fourth Affiliated Hospital of Xinjiang Medical University, Urumqi 83000I, Xinjiang, China

*These authors contributed equally to this work

\begin{abstract}
Adenosquamous carcinoma (ASC) of the lung, a biphasic malignant tumor arising from lung tissue, is a special subtype of non-small-cell lung cancer (NSCLC) with low incidence but high tendency of invasion and poor prognosis. ASC contains components of lung adenocarcinoma (AC) and lung squamous cell carcinoma (SCC). However, there is a remarkable difference between ASC and other NSCLCs in clinical features, suggesting that ASC is not a simple mixture of $\mathrm{AC}$ and $\mathrm{SCC}$, but is rather a more complex carcinoma with a unique molecular phenotype. At present, the research on ASC is still rare, mostly because of its complicated molecular mechanism and unclear pathological origin. The lack of cognition of ASC limits its early diagnosis and treatment, and a set of mature and effective treatment programs has not been proposed yet. In-depth study of the molecular characteristics and clinical features of ASC will not only help to better understand the scientific issues, including phenotype switching of lung cancer, the origin of tumor development, and tumor heterogeneity, but also contribute to the development of its individualized treatment. This review summarizes the recent studies concerning the clinicopathologic features and the molecular mechanisms of ASC to further facilitate the development of its individualized treatment.
\end{abstract}

Keywords: lung adenosquamous carcinoma, lung adenocarcinoma, lung squamous cell carcinoma, prognostic factor, clinicopathologic features

\section{Introduction}

Lung cancer is the most common cancer in the world and the most common cause of cancer deaths. Non-small-cell lung cancer (NSCLC) represents approximately 85\% of lung cancer cases and includes adenosquamous carcinoma (ASC), adenocarcinoma (AC), and squamous cell carcinoma (SCC). The incidence of ASC is relatively low and accounts for only $0.4 \%-4 \%$ of all lung carcinomas. ${ }^{1,2}$ Prior to 1999 , the definition of ASC was unclear as it was only defined as a mixture of SCC and AC. ${ }^{3}$ In 1999, the WHO defined ASC as a carcinoma containing components of both SCC and AC with each component constituting at least $10 \%$ of the tumor. ${ }^{4}$ However, this definition is still debated by some researchers. ${ }^{5}$ In general, the survival rates of ASC patients are worse than other NSCLC patients, despite surgical resection in early stage of disease compared to stage-matched NSCLC cases. ${ }^{6}$ Indeed, operation-based comprehensive treatment approach is currently applied to treat ASC, but the curative effect has not reached satisfaction. The criteria for diagnosis of ASC therefore may not be uniformly applied, leading to variation in the reported incidence and clinical and radiologic features. This review aims to summarize the current findings of the clinicopathologic features and the molecular mechanisms of ASC to further facilitate the development of its individualized treatment. 


\section{Clinicopathologic features of ASC Clinical features of ASC}

ASC is a relatively rare tumor with strong aggressiveness and poor prognosis. Indeed, a retrospective cohort study of patients who underwent lung resection showed that the postoperative cumulative 5-year survival rate of patients with ASC was significantly lower compared with the survival rate of patients with other types of carcinomas $(6.2 \%$ vs $41.5 \%))^{7}$ Furthermore, other cohort studies in $\operatorname{Japan}^{5}$ and France $^{8}$ confirmed that the 5-year survival rate of ASC is lower in all clinical or pathological stages compared with $\mathrm{AC}$ and SCC.

Even though ASC shares some clinical features with other NSCLCs, it has its own characteristics with a higher frequency of lymph node metastasis probably due to its rapid progression, aggressiveness, and early-stage metastasis. ${ }^{9}$ Moreover, a study showed that ASC patients had larger size of tumors, high tendency of vessel/nerve/lymphangion invasion, and more frequent ipsilateral multiple nodules than SCC and AC patients, with approximately half of the patients presenting large undifferentiated cells. ${ }^{8}$ On a demographic level, several studies have shown that ASC is more prevalent in male smokers who are over the age of 65 years old. ${ }^{5,10,11}$ Furthermore, Wang et al analyzed a large cohort of patients $(4,245$ with ASC, 124,253 with AC, and 74,710 with SCC) and found that while age, sex ratio, race, year of diagnosis, and tumor size of ASC patients were similar to those of AC and SCC patients, tumor grade was higher and prevalence of nodal metastasis was lower in ASC patients compared to AC and SCC patients. ${ }^{12} \mathrm{ASC}$ is more susceptible to tumor invasion and early metastasis and has a worse prognosis than AC and SCC. Therefore, patients at early stage of ASC may require more effective treatment and adjuvant chemotherapy.

Two studies including 104 and 205 patients with ASC, respectively, showed that, similar to other NSCLCs, the overall survival (OS) and disease-free survival (DFS) in ASC were associated with structural components, TNM stage, and adjuvant chemotherapy. ${ }^{13,14}$ They also demonstrated that the serum level of CA12-5 is another important prognostic factor and postoperative platinum-based chemotherapy improves survival in ASC patients. Moreover, ASC patients with balanced adenomatous and squamous component were associated with a better prognosis than those with one predominant component, $\mathrm{AC}$ or $\mathrm{SSC},{ }^{11,13,14}$ and this result is in line with Mordant et al's study. ${ }^{8}$ However, other studies presented contradictory findings and suggested that the patients with a balanced structure have the shortest survival time, and patients with more adenomatous components or with more squamous components have a longer survival time, which may be related to the use of targeted drugs, tumor tissue composition of different proportions, and small number of cases. ${ }^{15,16}$

Overall, ASC is a tumor with strong aggressiveness and its prognosis is poorer than AC and SCC.

\section{Imaging diagnosis features}

Lee et al studied the computed tomography (CT) and fluorodeoxyglucose positron emission tomography imaging and clinicopathologic features of 26 ASC patients. ${ }^{9}$ They found that $80 \%$ of the tumors were located in the peripheral lung field, and all the imaging features of the tumor were of low density, with mixed composition and higher standardized intake, which was closely related to the poor prognosis of the tumor. Moreover, Yu et al analyzed 29 ASC cases with enhanced CT imaging features and found that the tumor was located in the lung field and the center of the ratio was 72:28, mixed mass and unitary mass ratio was 69:31, and peripheral tumor manifested a higher proportion of intratumoral necrosis and uneven reinforcement performance. ${ }^{17}$ However, $\mathrm{Wu}$ et al suggested that the imaging findings of ASC were not specific. ${ }^{18}$ Indeed, the proportion of the two different pathological components cannot be differentiated from the imaging, and the relatively obvious features are limited to the majority of solid masses located in the peripheral lung fields. In general, the imaging findings of ASC can suggest some characteristics, but no obvious feature to differentiate from AC and SCC has been detected.

\section{Pathological diagnosis of ASC}

ASC is more difficult to be diagnosed than AC and SCC, and the accuracy of pathological diagnosis by cytology or tissue puncture is low. Indeed, in a retrospective study of 13 patients with ASC confirmed by pathology, preoperative pathological diagnosis was SCC in eight cases, AC in one case, and ASC in only four cases. ${ }^{19}$ Another study has also shown the difficulty involved in the diagnosis of ASC: a case report with the final diagnosis of ASC showed that the result of cytological examination of pleural effusion was NSCLC, and the result of immunohistochemistry was AC, but bronchial lavage and tracheal biopsy hinted SCC. ${ }^{20}$ The authors highlighted that the discovery of SCC is an important clinical feature to avoid the use of antiangiogenic monoclonal antibodies, thereby reducing the risk of treatment-related bleeding. 
In general, ASC contains two types of components which should be determined by H\&E staining or immunohistochemistry.

It should be noted that ASC contains different pathological subtypes, and that the heterogeneity of the resulting tumors adds to the difficulty of diagnosis. Indeed, Yamatani et al found that in 30 ASC cases, eight had more unique histopathological features, including a third component mucinous epidermoid tumor-like component, and basal immunohistochemical examination showed a characteristic p63 staining. ${ }^{21}$ ASC can be further subdivided into four subgroups, TTF1-/+ and p63-/+, by immunohistochemical staining of TTF1 and p63 cells.

The transfer behavior of ASC is a unique feature and may result from the two different pathological components, but further studies are needed to confirm these results. A study has reported that an ASC patient first relapsed with a pure squamous cell tumor in the brain, and surgery and radiotherapy rendered him disease-free. ${ }^{22}$ However, 18 months later, recurrence of solitary pure AC occurred in the lungs, and it was found that the primary tumor and the two recurrences shared the molecular features of EGFR with exon 19 del type. EGFR tyrosine kinase inhibitor (TKI) was not used during the treatment, which removed the possibility of histological-type changes induced by small-molecule TKIs. In another study, an ASC patient underwent cystic brainstem metastasis after operation. The puncture pathology showed that the cystic metastasis exhibited a good differentiation of AC-like constituents and a small amount of heterogeneous SCC. ${ }^{23}$ The stereotactic aspiration combined with Gamma Knife radiosurgery was used to treat the brainstem metastasis which originated from ASC.

\section{Advances in molecular research of ASC$$
\text { Special composition and molecular }
$$$$
\text { phenotype }
$$

The WHO has only defined ASC as a mixture of AC and $\mathrm{SSC}$, but it is unclear whether ASC is a simple mixture of these two components or it has a more complex and unique molecular phenotype.

At the gene level, several studies have demonstrated that ASC patients present mutations in two genes, EGFR and KRAS, in both components. ${ }^{24-30}$ Studies found that biopsy of pure SCC lacked the EGFR/KRAS mutations and the detection of these mutations in small samples diagnosed as SCC could be a result of incomplete sampling of ASC..$^{27,31}$
The mutation rate of EGFR/KRAS was somewhat similar to $\mathrm{AC}$, and the mutation often simultaneously existed in both AC and SCC. Another study of Western patients with ASC discovered that although the EGFR mutation rate in ASC patients is similar to that of AC patients, the KRAS mutations are rare. ${ }^{25}$

At the transcriptional level, a study by Bastide et al analyzed the differences between AC, SSC, and ASC in primary rat lung carcinoma. ${ }^{32}$ This study compared five AC, five SCC, and nine ASC cases and found that that the expression of 72 genes could be used to differentiate AC from SCC, 40 genes to differentiate SCC from ASC, and 39 genes to differentiate AC from ASC. Furthermore, the gene expression seemed to reflect the mixture of AC and SCC in ASC: expression of some genes of ASC was similar to that of AC, while other genes had a similar expression as genes of SCC. However, ASC also showed a unique expression of some tumor-related genes, especially neuroendocrine differentiation and ERK-related pathway genes.

At the protein level, several studies have demonstrated that $\mathrm{AC}$ and SCC differed in their expression of thymidine synthase (TS) with a higher expression in SCC. ${ }^{33-35}$ This enzyme is important in de novo DNA synthesis, and its inhibition by the drug pemetrexed leads to arrested cell proliferation. However, high expression of TS in tumors was shown to make the tumor resistant to pemetrexed. ${ }^{36}$ Shu et al analyzed the expression of TS in ASC patients and found that the expression was high, higher than in $\mathrm{AC}$ patients and similar to the level found in SCC patients. This finding indicated that pemetrexed might not be the best choice of treatment. ${ }^{24}$

So far, it is considered that ASC has its own biological behavior, and it is not a simple mixture of two kinds of tissue components.

\section{Origin speculations}

There are currently three theories hypothesizing the origin of ASC: 1) the theory of "collision" between $\mathrm{AC}$ and SCC, 2) the theory of transition between AC and SCC and 3) "common precursors" theory.

The collision theory supports the idea that ASC is the product of collision between separate independent foci of AC and SCC. One study in an animal model seemed to suggest that AC and SCC can be spatially separated into small nodules but can collide and form larger nodules of both components. ${ }^{37}$ Furthermore, Yazawa et al reported one case of primary "collision" lung cancer with two closely 
linked nodules. ${ }^{38}$ One nodule contained well-differentiated AC-like constituents and poor-differentiated SCC, and where the loss of heterozygosity of AR and PGK-1 genes can be detected, while the other nodule was closely connected with the former, and mainly included large-cell neuroendocrinelike tumor components with partial squamous cell-like differentiation and contained alleles for both AR and PGK-1. The "collision-type" tumor in this report was more typical, the histological morphology of the two nodules was very different, and the detection of clonal heterozygosity deletion (loss of heterozygosity, LOH) further confirmed that the two nodules were not single cloned sources.

Another theory of the ASC origin deals with transition between AC and SCC. Tsuhako et al found that human papillomavirus (HPV) DNA expression was present in the tissue of regional ASC patients. ${ }^{39}$ The AC-like cells near the SCC-like component were larger and were positive for HPV, high-molecular weight cytokeratin, and skin protein involucrin. These results suggested that AC-like components were transitioning to SCC. Another study by Kanazawa et al analyzed the changes of p53 and KRAS genes, 9p21 and 9q31-32 chromosome abnormalities, and cellular immunohistochemistry in 12 ASC patients. ${ }^{40}$ They found overexpression of p53 gene in both components of ASC and in the intermediate transitional tissue. 9p21 and 9q31-32 chromosome abnormalities also existed in the two components. Moreover, the AC-like component showed a higher expression of SCC-related antigen and expression of mucin-1 was also significantly reduced compared to pure AC. The authors suggested that ASC was in the process of metastasis from single-cell carcinoma to AC.

The third theory is that ASC originated from common precursors, but differentiated into different tissue forms in the course of disease. At present, many studies support this theory through the analysis of chromosome linkage polymorphism markers, LOH detection, or EGFR/RAS mutation characteristics. Niho et al analyzed the clonality of the two components of ASC on an X-chromosome-linked polymorphic marker, HUMARA, in four female patients. ${ }^{41,42}$ They discovered that AC and SCC components showed identical monoclonal patterns, and it was inferred that the two components originated from the same precursor.

SCC is generally believed to originate from the upper airway basal cells and express cytokeratin-5 (CK5+), and $\mathrm{AC}$ is believed to originate from stem cells at the junction of the bronchial alveoli and express cytokeratin-7 (CK7+). Mather et al isolated the ASC tumor stem cells co-expressing cytokeratin-5 and cytokeratin-7 (CK5+ CK7+), cultured them in vitro, and grafted these cells into immune-deficient mice. ${ }^{43}$ They showed that these cells could successfully differentiate into SCC- and AC-like components in mice, and these cells had the potential to differentiate into tracheal, alveolar, or neuroendocrine cells. ASC is therefore thought to originate from the primordial lung stem cells, and not from the basal cells or the stem cells at the bronchial alveolar junction.

To further clarify the molecular features of different cellular components in ASC, a study integrated the use of next-generation sequencing, fluorescence in situ hybridization (FISH), and laser micro-cutting technique to characterize the tissue samples obtained from 16 Caucasian ASC patients. ${ }^{44}$ The results showed that several molecular mutant phenotypes were similar between the two components. The prevalence of EGFR mutations and mutations in the PI3K signaling pathway was found to be significantly higher in ASC patients (30\% and $25 \%$, respectively) compared to AC and SCC patients, while the KRAS mutation was less prevalent. In order to better understand the tumor heterogeneity, Gerlinger et al hypothesized that the intratumoral gene mutations of monoclonal origin can be divided into "trunk" and "branch" types. ${ }^{45}$ They found that the driving mutant genes were concentrated in the "trunk", and the "branch" appeared in the later stage of the tumor and contained a number of passenger mutant genes. These results suggested that the different tissue types of ASC had the same origin, rather than being formed by "collisions" of two different tissue types. ${ }^{44}$ Furthermore, a case report of esophageal ASC by van Rees et al showed that the AC and SC components shared the loss of the same alleles at different markers on different chromosomes and contained the same missense mutation in $\mathrm{p} 53^{46}$, supporting the latter origin hypothesis.

\section{Treatment and prognosis of ASC}

Surgery is the first choice and main treatment of ASC, and the surgical procedure is similar to other NSCLC treatments. Although the postoperative survival rate of patients is improved, the prognosis is still not satisfactory compared with $\mathrm{AC}$ and $\mathrm{SC}$. Maeda et al conducted a retrospective cohort study including 114 cases of ASC (2.4\%), 2,993 cases of $\mathrm{AC}(64.2 \%)$, and 1,369 cases of SCC (29.3\%) who underwent surgical treatment. ${ }^{5}$ The results showed that the 5-year survival rate was $23.3 \%$ for ASC patients, 58\% for AC patients, and $40.8 \%$ for SCC patients, while in pIA stage the survival rates were $42.0 \%, 81.8 \%$, and $63.4 \%$, respectively. Therefore, effective postoperative adjuvant chemotherapy is needed for the patients at early stage of ASC. 
Postoperative platinum-based chemotherapy can improve patient survival and reduce the risk of distant metastases in patients with resectable ASC. ${ }^{47,48}$ However, different components of NSCLCs respond differently to chemotherapy regimens. AC may respond more to pemetrexed plus cisplatin, whereas SCC may be more responsive to gemcitabine plus cisplatin ${ }^{49}$ whether the efficacy of the chemotherapy regimen of ASC depends on the distribution of its constituents has not yet been determined. EGFR-TKIs are the first-line chemotherapy regimen for EGFR-mutated NSCLCs. ${ }^{50}$ Fan et al showed that in a total of 27 patients with EGFR mutations treated with EGFR-TKIs, the disease control rate was 74.1\% (20/27) and OS was 39 months (95\% CI: 12.9-17.1). The results showed that ASC responded well to TKI treatment, but further clinical researches are still needed to confirm the benefits of TKI vs simple chemotherapy. According to the recent progress in the treatment of lung cancer, distinction between AC and SCC is of great significance to determine the best treatment agent, including pemetrexed, TKIs, monoclonal antibody against vascular endothelial growth factor, and crizotinib. All of these drugs, as well as antiangiogenic therapy, PD-1 immunotherapy, and other molecular targeted therapy, are under clinical studies for treatment of ERBB2-, KRAS-, PIK3CA-, and AKT1-mutated ASC. The clinical results of these studies will help to improve the choice of treatment of ASC.

The prognosis of ASC is related to pathological staging, surgical treatment, choice of adjuvant chemotherapy regimen, and the basic condition of patients. Early diagnosis of ASC will improve the prognosis to a large extent. The role of neutrophil percentage, platelet count, platelet percentage, and fibrinogen content in the prognosis of NSCLC has been reported in a number of studies; however, a recent research found that the combined results were closely correlated with the decline of DFS and OS in ASC. ${ }^{51}$ In addition, studies indicated that serum level of CA125, high expression of preoperative NSE, thyroid transcription factor-1, and MET amplification were related to the prognosis of ASC, but the highly convenient and effective prognostic indicators are still being explored. ${ }^{13,52,53}$

\section{Conclusion and outlook}

At present, the individualized treatment of ASC does not involve a unique treatment strategy. The treatment of the early ASC mainly includes surgery, supplemented by chemotherapy and other comprehensive treatment with poor efficiency. Hence, more studies are needed for further exploration. ASC is difficult to be diagnosed accurately through small-tissue biopsy. Current studies have shown that the mutations in the two components of ASC are similar to EGFR-driven mutations, and raise the question that whether the patients who have been diagnosed with SCC by smalltissue biopsy should be further tested for EGFR/KRAS mutations. Among the different biopsy techniques, liquid biopsy may be a better method for target gene detection in advanced ASC. Use of thymidine synthase as a predictive marker to evaluate the therapeutic effect of metoprolol is still in debate. Additionally, ASC patients have two components, $\mathrm{AC}$ and SCC, which leads to difference in clinical treatment results. Prognosis of ASC treated with gene therapy targeting two different pathway molecules might be inferior to $\mathrm{AC}$ or SCC. Different proportions of the two components may lead to different prognosis and might indicate the need to identify more controllable targets and pathways. However, more evidence is warranted to confirm the current hypothesis to improve the treatment effect and prognosis of ASC.

\section{Acknowledgment}

This work was supported by the National Natural Science Foundation of China (grant number: 81573003).

\section{Disclosure}

The authors report no conflicts of interest in this work.

\section{References}

1. Siegel R, Naishadham D, Jemal A. Cancer statistics, 2013. CA Cancer J Clin. 2013;63(1):11-30.

2. Shundo Y, Takahashi T, Itaya T, et al. Clinical study of forty-two patients who underwent resection for pulmonary adenosquamous carcinoma. Kyobu Geka. 2011;64(10):871876-876879.

3. The Japan Lung Cancer Society. General Rules for Clinical and Pathological Record of Lung Cancer. 3rd ed. Tokyo: Kanehara; 1987.

4. Teng XD. World Health Organization classification of tumours, pathology and genetics of tumours of the lung. Zhonghua Bing Li Xue Za Zhi. 2005;34(8):544-546.

5. Maeda H, Matsumura A, Kawabata T, et al. Adenosquamous carcinoma of the lung: surgical results as compared with squamous cell and adenocarcinoma cases. Eur J Cardiothorac Surg. 2012;41(2):357-361.

6. Filosso PL, Ruffini E, Asioli S, et al. Adenosquamous lung carcinomas: a histologic subtype with poor prognosis. Lung Cancer. 2011;74(1): 25-29.

7. Nakagawa K, Yasumitu T, Fukuhara K, Shiono H, Kikui M. Poor prognosis after lung resection for patients with adenosquamous carcinoma of the lung. Ann Thorac Surg. 2003;75(6):1740-1744.

8. Mordant P, Grand B, Cazes A, et al. Adenosquamous carcinoma of the lung: surgical management, pathologic characteristics, and prognostic implications. Ann Thorac Surg. 2013;95(4):1189-1195.

9. Lee Y, Chung JH, Kim SE, Kim TJ, Lee KW. Adenosquamous carcinoma of the lung: CT, FDG PET, and clinicopathologic findings. Clin Nucl Med. 2014;39(2):107-112.

10. Watanabe Y, Tsuta K, Kusumoto M, et al. Clinicopathologic features and computed tomographic findings of 52 surgically resected adenosquamous carcinomas of the lung. Ann Thorac Surg. 2014;97(1): $245-251$. 
11. Gawrychowski J, Bruliński K, Malinowski E, Papla B. Prognosis and survival after radical resection of primary adenosquamous lung carcinoma. Eur J Cardiothorac Surg. 2005;27(4):686-692.

12. Wang J, Lian B, Ye L, Hu J, Song Y. Clinicopathological characteristics and survival outcomes in adenosquamous carcinoma of the lung: a population-based study from the SEER database. Oncotarget. 2018; 9(8):8133-8146.

13. Lian S, Huang Y, Yang H, Zhao H. Serum carbohydrate antigen 12-5 level enhances the prognostic value in primary adenosquamous carcinoma of the lung: a two-institutional experience. Interact Cardiovasc Thorac Surg. 2016;22(4):419-424.

14. Zhao H, Yang H, Yao F, et al. Improved survival associated with a balanced structure between adenomatous and squamous components in patients with adenosquamous carcinoma of the lung. Eur J Surg Oncol. 2016;42(11):1699-1706.

15. Guo Y, Jia L, Shao GG, et al. Clinicopathological characteristics and prognosis of patients with adenosquamous lung carcinoma. J Huazhong Univ Sci Technolog Med Sci. 2015;35(3):350-355.

16. Ruffini E, Rena O, Oliaro A, et al. Lung tumors with mixed histologic pattern. Clinico-pathologic characteristics and prognostic significance. Eur J Cardiothorac Surg. 2002;22(5):701-707.

17. Yu JQ, Yang ZG, Austin JH, Guo YK, Zhang SF. Adenosquamous carcinoma of the lung: CT-pathological correlation. Clin Radiol. 2005; 60(3):364-369.

18. Wu N, Lu N, Chen Y. Adenosquamous carcinoma of the lung. A radiologic and pathologic study. Zhonghua Zhong Liu Za Zhi. 1997;19(6): 434-436.

19. Damadoğlu E, Aybatli A, Yalçinsoy M, et al. Adenosquamous carcinoma of the lung (an analysis of 13 cases). Tuberk Toraks. 2005;53(2): 161-166.

20. Shelton DA, Rana DN, Holbrook M, Taylor P, Bailey S. Adenosquamous carcinoma of the lung diagnosed by cytology? A diagnostic dilemma. Diagn Cytopathol. 2012;40(9):830-833.

21. Yamatani C, Abe M, Shimoji M, et al. Pulmonary adenosquamous carcinoma with mucoepidermoid carcinoma-like component with characteristic p63 staining pattern: either a novel subtype originating from bronchial epithelium or variant mucoepidermoid carcinoma. Lung Cancer. 2014;84(1):45-50.

22. Burkart J, Shilo K, Zhao W, Ozkan E, Ajam A, Otterson GA. Metastatic Squamous Cell Carcinoma Component from an Adenosquamous Carcinoma of the Lung with Identical Epidermal Growth Factor Receptor Mutations. Case Rep Pulmonol. 2015;2015:283875.

23. Du C, Li Z, Wang Z, Wang L, Tian YU. Stereotactic aspiration combined with gamma knife radiosurgery for the treatment of cystic brainstem metastasis originating from lung adenosquamous carcinoma: A case report. Oncol Lett. 2015;9(4):1607-1613.

24. Shu C, Cheng H, Wang A, et al. Thymidylate synthase expression and molecular alterations in adenosquamous carcinoma of the lung. Mod Pathol. 2013;26(2):239-246.

25. Tochigi N, Dacic S, Nikiforova M, Cieply KM, Yousem SA. Adenosquamous carcinoma of the lung: a microdissection study of KRAS and EGFR mutational and amplification status in a western patient population. Am J Clin Pathol. 2011;135(5):783-789.

26. Jia XL, Chen G. EGFR and KRAS mutations in Chinese patients with adenosquamous carcinoma of the lung. Lung Cancer. 2011;74(3): 396-400.

27. Kang SM, Kang HJ, Shin JH, et al. Identical epidermal growth factor receptor mutations in adenocarcinomatous and squamous cell carcinomatous components of adenosquamous carcinoma of the lung. Cancer. 2007;109(3):581-587.

28. Ohtsuka K, Ohnishi H, Fujiwara M, et al. Abnormalities of epidermal growth factor receptor in lung squamous-cell carcinomas, adenosquamous carcinomas, and large-cell carcinomas: tyrosine kinase domain mutations are not rare in tumors with an adenocarcinoma component. Cancer. 2007;109(4):741-750.

29. Toyooka S, Yatabe Y, Tokumo M, et al. Mutations of epidermal growth factor receptor and K-ras genes in adenosquamous carcinoma of the lung. Int J Cancer. 2006;118(6):1588-1590.
30. Sasaki H, Endo K, Takada M, et al. EGFR exon 20 insertion mutation in Japanese lung cancer. Lung Cancer. 2007;58(3):324-328.

31. Ladanyi M, Pao W. Lung adenocarcinoma: guiding EGFR-targeted therapy and beyond. Mod Pathol. 2008;21(Suppl 2):S16-S22.

32. Bastide K, Ugolin N, Levalois C, Bernaudin JF, Chevillard S. Are adenosquamous lung carcinomas a simple mix of adenocarcinomas and squamous cell carcinomas, or more complex at the molecular level? Lung Cancer. 2010;68(1):1-9.

33. Ceppi P, Volante M, Novello S, et al. ERCC1 and RRM1 gene expressions but not EGFR are predictive of shorter survival in advanced non-small-cell lung cancer treated with cisplatin and gemcitabine. Ann Oncol. 2006;17(12):1818-1825.

34. Sun JM, Han J, Ahn JS, Park K, Ahn MJ. Significance of thymidylate synthase and thyroid transcription factor 1 expression in patients with nonsquamous non-small cell lung cancer treated with pemetrexed-based chemotherapy. J Thorac Oncol. 2011;6(8):1392-1399.

35. Tanaka F, Wada H, Fukui Y, Fukushima M. Thymidylate synthase (TS) gene expression in primary lung cancer patients: a large-scale study in Japanese population. Ann Oncol. 2011;22(8):1791-1797.

36. Ozasa H, Oguri T, Uemura T, et al. Significance of thymidylate synthase for resistance to pemetrexed in lung cancer. Cancer Sci. 2010; 101(1):161-166.

37. Hammond WG, Tesluk H, Benfield JR. Histogenesis of adenosquamous bronchogenic carcinoma. Cancer Lett. 1995;96(2):163-168.

38. Yazawa $\mathrm{T}$, Ishii $\mathrm{H}$, Ito $\mathrm{T}$, et al. Colliding primary lung cancers of adenosquamous carcinoma and large cell neuroendocrine carcinoma. Pathol Int. 2003;53(1):58-65.

39. Tsuhako K, Nakazato I, Hirayasu T, Sunakawa H, Iwamasa T. Human papillomavirus DNA in adenosquamous carcinoma of the lung. J Clin Pathol. 1998;51(10):741-749.

40. Kanazawa H, Ebina M, Ino-Oka N, et al. Transition from squamous cell carcinoma to adenocarcinoma in adenosquamous carcinoma of the lung. Am J Pathol. 2000;156(4):1289-1298.

41. Beal JE, Olson R, Laubenstein L, et al. Dronabinol as a treatment for anorexia associated with weight loss in patients with AIDS. J Pain Symptom Manage. 1995;10(2):89-97.

42. Niho S, Yokose T, Kodama T, Nishiwaki Y, Mukai K. Clonal analysis of adenosquamous carcinoma of the lung. Jpn J Cancer Res. 1999;90(11): 1244-1247.

43. Mather JP, Roberts PE, Pan Z, et al. Isolation of cancer stem like cells from human adenosquamous carcinoma of the lung supports a monoclonal origin from a multipotential tissue stem cell. PLoS One. 2013; 8(12):e79456.

44. Vassella E, Langsch S, Dettmer MS, et al. Molecular profiling of lung adenosquamous carcinoma: hybrid or genuine type? Oncotarget. 2015;6(27):23905-23916

45. Gerlinger M, Rowan AJ, Horswell S, et al. Intratumor heterogeneity and branched evolution revealed by multiregion sequencing. $N$ Engl $J$ Med. 2012;366(10):883-892.

46. van Rees BP, Rouse RW, de Wit MJ, et al. Molecular evidence for the same clonal origin of both components of an adenosquamous Barrett carcinoma. Gastroenterology. 2002;122(3):784-788.

47. Kris MG, Gaspar LE, Chaft JE, et al. Adjuvant Systemic Therapy and Adjuvant Radiation Therapy for Stage I to IIIA Completely Resected Non-Small-Cell Lung Cancers: American Society of Clinical Oncology/ Cancer Care Ontario Clinical Practice Guideline Update. J Clin Oncol. 2017;35(25):2960-2974.

48. Ettinger DS, Wood DE, Akerley W, et al. NCCN Guidelines Insights: Non-Small Cell Lung Cancer, Version 4.2016. J Natl Compr Canc Netw. 2016;14(3):255-264.

49. Scagliotti GV, Parikh P, von Pawel J, et al. Phase III study comparing cisplatin plus gemcitabine with cisplatin plus pemetrexed in chemotherapy-naive patients with advanced-stage non-small-cell lung cancer. J Clin Oncol. 2008;26(21):3543-3551.

50. Fan L, Yang H, Yao F, et al. Clinical outcomes of epidermal growth factor receptor tyrosine kinase inhibitors in recurrent adenosquamous carcinoma of the lung after resection. Onco Targets Ther. 2017;10: 239-245. 
51. Wang YQ, Zhi QJ, Wang XY, Yue DS, Li K, Jiang RC. Prognostic value of combined platelet, fibrinogen, neutrophil to lymphocyte ratio and platelet to lymphocyte ratio in patients with lung adenosquamous cancer. Oncol Lett. 2017;14(4):4331-4338.

52. Yeung SF, Tong JHM, Law PPW, et al. Profiling of Oncogenic Driver Events in Lung Adenocarcinoma Revealed MET Mutation as Independent Prognostic Factor. J Thorac Oncol. 2015;10(9):1292-1300.
53. Zhi Q, Wang Y, Wang X, Yue D, Li K, Jiang R. Predictive and prognostic value of preoperative serum tumor markers in resectable adenosqamous lung carcinoma. Oncotarget. 2016;7(40):64798-64809.

\section{Publish your work in this journal}

OncoTargets and Therapy is an international, peer-reviewed, open access journal focusing on the pathological basis of all cancers, potential targets for therapy and treatment protocols employed to improve the management of cancer patients. The journal also focuses on the impact of management programs and new therapeutic agents and protocols on

\section{Dovepress}

patient perspectives such as quality of life, adherence and satisfaction. The manuscript management system is completely online and includes a very quick and fair peer-review system, which is all easy to use. Visit http://www.dovepress.com/testimonials.php to read real quotes from published authors.

Submit your manuscript here: http://www.dovepress.com/oncotargets-and-therapy-journal 